\title{
Repair with resource reserve
}

\author{
Valeriy Varnikov ${ }^{1, *}$ \\ ${ }^{1}$ Moscow State University of Civil Engineering, Yaroslavskoye sh. 26, Moscow, 129337, Russia
}

\begin{abstract}
The task of maintaining the operability of complex technical systems is one of the important tasks of ensuring the reliability in high-rise construction. It is solved at all stages of the life cycle of the device. Ensuring high availability of technical devices for use during the operational phase is the timely conduct of preventive measures and routine maintenance. To this end, the repair authorities have in their inventory material resources to replace the abandoned and end-of-life elements, as well as the production facilities necessary to perform the repair itself. One of the possible variants of this method of repair can be the creation and use of significant production capacities of repair enterprises that do not need material resources, and carry out repairs themselves. Another option may be a large range of spare parts, which will make an additional link repair company. The paper considers the issues of creating a rational organization of repair of complex technical systems. At the same time, one of the important practical tasks is the task of determining the rational relationship between the production capacities of repair enterprises and the amount of reserves needed to perform repairs. The development of the initial theoretical provisions on the rationale for the rational correlation of the volumes of spare parts and production capacities of repair enterprises to the effective use of reserves is devoted to this article.
\end{abstract}

\section{Introduction}

Requirements for high availability of complex technical systems force specialists to create inventories in the form of separate parts, assemblies, sets and whole complex objects of technical devices. Together with the repair bodies, reserves in the form of exchange funds and spare parts make it possible to increase the readiness of the technical systems in operation. One of the important practical tasks is the task of determining the rational relationship between the production capacity of repair bodies and the volume of the corresponding reserves. Really, it is possible to create so powerful repair bodies that the stocks of the samples used for replacement directed for repair, it is not required. It is possible and the opposite solution, when there are only stocks of technical equipment, and the repair bodies are either not available, or are used mainly to perform erection work to replace the failed technical means. This practical problem must be proved theoretically.

\section{Materials and Methods}

\footnotetext{
*Corresponding author: vvsmgsu@rambler.ru
} 
The model is based on the Markov [1] processes of queuing theory. During the repair, the repair body "P" receives a stream of applications with an intensity $\omega=1 / \mathrm{Tcp}$, where Tcp is the average time between applications for repairs. Then, the working capacity and resource of the technical systems being repaired are restored with an intensity $\mu$ equal to $\mu=1 / \mathrm{Tb}$, where $\mathrm{Tb}$ is the average recovery time. Thus, the state of the object being repaired can be represented as the state of its readiness "G", and the repair state "R" [2]. The interrelation between these states is represented in the form of Fig. 1

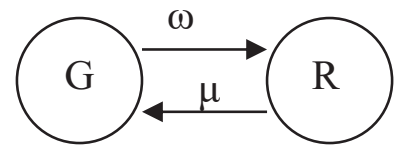

Fig.1 The relationship between the "G" and "R"

To study the state of an object, the process of recovery is considered as a mass service process.

Let us find the analytical relationship between Pr and the parameters $\omega$ and $\mu$. To do this, we formulate a system of differential equations. In the case under consideration, the two quantities $\mathrm{Pr}$ and $\mathrm{Pp}$ are unknown. Therefore, for a complete description of the queuing system in question it is necessary to have two equations. In accordance with the Kolmogorov rule, [3] we have

$$
\begin{aligned}
& \frac{d R_{g}}{d t}=-\omega R_{g}+\mu R_{r} \\
& \frac{d R_{r}}{d t}=-\mu R_{r}+\omega R_{g} \\
& R_{g}+R_{r}=1
\end{aligned}
$$

The solution of this system of equations is possible by numerical methods or with the help of a stationary (method), equating the derivatives with respect to $t$ to zero. To find the stationary solution, we use the Cramer method [4 ].

So

$$
R_{g}=\frac{\Delta g}{\Delta}
$$

where

$$
\begin{aligned}
& \Delta=\left|\begin{array}{cc}
-\omega & \mu \\
1 & 1
\end{array}\right|=\mu+\omega \\
& \Delta_{g}=\left|\begin{array}{cc}
0 & \mu \\
1 & 1
\end{array}\right|=\mu
\end{aligned}
$$

The elements of the matrix are the coefficients of the system of equations (2)

$$
\begin{array}{ll}
\text { Consequently, } & R_{g}=\frac{\mu}{\mu+\omega}=\frac{1}{1+K} \\
\text { where } & \mathrm{K}=\frac{\omega}{\mu}
\end{array}
$$

The probability of finding an object in the ready state at any time - the availability factor of the object is a function of $\mathrm{K}$. The dependence of $\operatorname{Pr}(\mathrm{K})$ is shown in Table 1

Table 1. The dependence $\mathrm{Pg}_{\mathrm{g}}$ from $\mathrm{K}$

\begin{tabular}{c|c|c|c|c|c|c|c}
\hline $\mathrm{K}$ & 0 & 0,1 & 0,3 & 0,5 & 1,0 & 3,0 & 5,0 \\
\hline $\mathrm{R}_{\mathrm{g}}$ & 1,0 & 0,9 & 0,7 & 0,6 & 0,5 & 0,2 & 0,1 \\
\hline
\end{tabular}


From Table 1 it follows that the availability factor is the greater, the greater the productivity $\mu$ in relation to the intensity of repair orders $\omega$. Thus, for $K=1 \operatorname{Pr}=0.5$. Increasing $\mu$ with respect to $\omega$ by a factor of 2 , we obtain $K=0.5$ and $\operatorname{Pr}(0.5)=0.67$.

\subsection{Model of repair in the presence of a reserve of resources in a substitute fund}

The case in question refers to a situation where there are no stocks of objects. In this case, the throughput of the repair body K must be determined from condition (6). When objects return to repair with intensity $\omega 1$, spare objects with intensity $\lambda_{3}$ become ready. At the same time, the efficiency of the work can be increased by returning to the state of readiness of the $\rho$-th part of the repaired objects. In this case, the intensity of the transition of objects from the state "P" to the state "G" is equal to $\mu_{1} \rho$. The state of the stock "Z" is replenished with that part of the repaired objects which are not returned to the ready state. The intensity of the transition from the state "R" to the state "3", therefore, is equal to $\mu 1(1-\rho)$. The system of differential equations for this case has the form

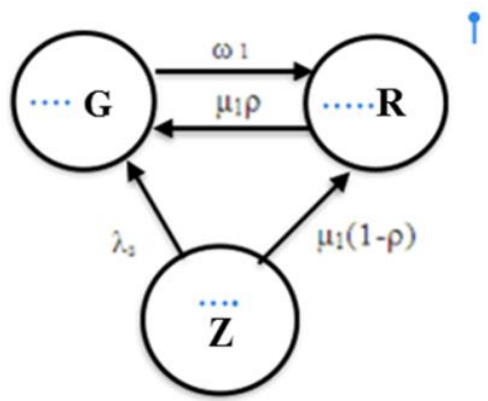

Fig.2. Influence of stocks on the work of remorgants

$$
\mathrm{K}=\frac{1-\rho_{g}}{\rho_{g}}
$$

and productivity. (Production capacity) of repair bodies

$$
\mu=\omega \frac{R_{g}}{1-R_{g}}
$$

In order to have values of the availability indicator close to unity, the productivity $\mu$ should be significantly greater than unity, the intensity of the requests for repairs. This, for $\operatorname{Pr}=0.6$ performance $\mu=1,5 \omega$.

One of the ways to reduce the capacity of $\mu$ is to create a reserve of objects that could be used to replace those that went into repair. To assess the effect of reserves on the performance of repair organs, consider the model in Fig. 2. In contrast to the model of Fig. 1 here we have the state of stock objects " 3 ". When objects return to repair with an intensity of $\omega 1$, all spare objects with intensity $\lambda_{3}$ are transferred to the ready state.

Simultaneously, the efficiency of work can be increased by returning to the ready state of the r-th part of the repaired objects. In this case, the intensity of the transition of the object from the state "P" to the state "G" is equal to $\mu 1 \rho$. The state of the stock " 3 " is replenished with that part of the repaired objects that are not returned to the ready state. The intensity of the transition from the state "P" to the state " 3 ", therefore, is equal to $\mu 1(1-\rho)$. The system of differential equations for this case has the form [5]

$$
\frac{d R_{g}}{d t}=-\omega_{1} R_{g}+\mu_{1} \rho R_{\rho}+\lambda_{z} R_{z}
$$




$$
\begin{gathered}
\frac{d R_{r}}{d t}=-\mu_{1} R_{r}+\omega_{i} P_{g} \\
\frac{d R_{3}}{d t}=-\lambda_{z} R_{z}+\mu_{i}(1-\rho) \rho_{\rho} ; \\
R_{g}+R_{z}+R_{r}=1
\end{gathered}
$$

Taking into account that the readiness indicator of $\operatorname{Pr}_{3}$ in this case should be considered as an indicator of system redundancy

Hence we have

$$
\begin{aligned}
& \rho_{\mathrm{gz}}=\rho_{\mathrm{g}}+\rho_{\rho} \\
& P_{g z}=\frac{\dot{\Delta}_{g}}{\Delta}+\frac{\Delta_{z}}{\Delta}=\frac{1}{1+\frac{K_{1}}{1+q(1-\rho)}} \\
& \dot{\mathrm{R}}_{\mathrm{g}}=\frac{1}{1+\mathrm{q}(1-\rho)+\mathrm{K}_{1}} \\
& \mathrm{P}_{\mathrm{z}}=\frac{1}{1+\frac{1}{\mathrm{q}}\left(\frac{1}{1-\rho}+\frac{\mathrm{K}}{1-\rho}\right)} \\
& K_{1}=\frac{\omega_{1}}{\mu_{1}} ; q=\frac{\omega_{1}}{\lambda_{z}} ; \frac{\lambda_{\mathrm{z}}}{\mu_{1}}=\frac{\mathrm{K}}{\mathrm{q}} \\
& \mathrm{K}_{1}=[1+\mathrm{q}(1-\rho)] \frac{1-\mathrm{P}_{\mathrm{gz}}}{\mathrm{P}_{\mathrm{gz}}}
\end{aligned}
$$

The required intensity of the use of reserves in the process of repair is determined by the expression

$$
\lambda_{z}=\frac{\left(1-\rho_{g z}\right)(1-\rho)}{\rho_{g z}\left(\frac{1-R g z}{\omega_{1} P g z}-\frac{1}{\mu_{1}}\right)}
$$

\section{Results}

Expressions (11) - (14), allow to estimate the effectiveness of the introduction of the reserve during repair [6]. The indicator of the effectiveness of the stock in this case is the ratio $\mu / \mu_{1}$

We can estimate how many times the performance of the remorgants can be reduced if you enter a stock of repair objects. Obviously, the introduction of a stock is more effective the more the productivity $\mu$ is relation to the productivity $\mu 1$. To establish the quantitative dependence $\mu, \mu_{1}, \lambda z, \omega, \omega_{1}, R_{g}$ we will proceed from , that the indicator of readiness of the system with stocks Pr should be greater than the readiness indicator in the absence of reserves

$$
\mathrm{R}_{\mathrm{Gz}} \geq \mathrm{R}_{\mathrm{G}}
$$

Substituting the value of the quantities into inequality (17), we obtain

Consequently

Hence the ratio of throughputs

$$
\frac{1}{1+\frac{K_{1}}{1+q(1-\rho)}} \geq \frac{1}{1+K}
$$




$$
\frac{\mu}{\mu_{1}}>\frac{\omega}{\omega_{1}}[1+q(1-\rho)] \approx 1+q(1-\rho)
$$

The dependence $\frac{\mu}{\mu_{1}}$ as function of $\mathrm{q}$ and $\rho$ is presented in Table. 2. It characterizes the gain in the productivity of repair bodies when stock is introduced. From Table. 2 it follows that the introduction of reserves corresponding to the ratio $\frac{\omega_{1}}{\lambda_{3}}=1$, i.e. volume $m_{3}=\omega_{1}, t$ at $\rho=$ 0 provides a performance gain repair bodies 2 times.

Table 2. The performance of repair bodies in the presence of stocks

\begin{tabular}{|c|c|c|c|c|c|c|c|c|c|}
\hline $\mathrm{q} \rho$ & 0 & 0,1 & 0,2 & 0,3 & 0,4 & 0,5 & 1,0 & 2,0 & 3,0 \\
\hline 0 & 1 & 1,1 & 1,2 & 1,3 & 1,4 & 1,5 & 2,0 & 3,0 & 4,0 \\
\hline 0,5 & 1 & 1,05 & 1,1 & 1,15 & 1,2 & 1,25 & 1,5 & 2,0 & 2,5 \\
\hline 0,9 & 1 & 1,01 & 1,02 & 1,03 & 1,04 & 1,05 & 1,1 & 1,2 & 1,3 \\
\hline
\end{tabular}

\subsection{Winning the index object readiness}

We will estimate under what conditions the introduction of reserves is expedient. It follows from the condition (17) that the gain in the readiness index Pr, when the stock of objects is introduced, is determined by the inequality $\rho_{g z}>\rho_{g} \quad \eta=\frac{\rho_{g z}}{\rho_{g}}>1$,

Option 1. Let's consider two variants of functioning of system of repair at presence of a stock option 1.

The repair system $[7,8]$ provides for the restoration of objects with an intensity of $\mu_{1}$ equal to the intensity of receipt of repair orders $\omega_{1,},\left(\mu_{1}=\omega_{1}\right)$, therefore, the parameter $K_{1}=1$.

The samples sent for repair are replaced with spare samples with intensity $\lambda_{3}$. Repaired samples replenish the stock $(\rho=0)$. In order to estimate the influence of the intensity of the expenditure of reserves on the efficiency of recovery, we determine $\mathrm{P}_{\mathrm{g} 3}, \mathrm{P}_{\mathrm{g}}, \mathrm{P}_{3}$, and $\eta$. The results of the calculations are presented in Table 3.

Table 3. The results of the calculation of the readiness and efficiency of stocks during repair

\begin{tabular}{|c|c|c|c|c|c|c|c|c|c|c|}
\hline & 0 & 0,1 & 0,2 & 0,3 & 0,5 & 1 & 2 & 3 & 5 & 10 \\
\hline$\dot{\mathrm{R}}_{\mathrm{g}_{1}}$ & 0,5 & 0,48 & 0,45 & 0,44 & 0,40 & 0,33 & 0,25 & 0,20 & 0,14 & 0,08 \\
\hline$R_{z_{1}}$ & 0 & 0,05 & 0,09 & 0,13 & 0,20 & 0,33 & 0,50 & 0,60 & 0,71 & 0,83 \\
\hline$R_{g z_{1}}$ & 0,50 & 0,52 & 0,545 & 0,57 & 0,60 & 0,67 & 0,75 & 0,80 & 0,86 & 0,92 \\
\hline$\eta_{1}$ & 0,5 & 0,57 & 0,66 & 0,73 & 0,90 & 1,34 & 2,25 & 3,20 & 5,13 & 10,06 \\
\hline
\end{tabular}

From Table 3 it follows that in this version the efficiency $\eta$ becomes greater than 1 for the parameter values $q=\frac{\omega_{1}}{\lambda_{z}}>2 / 3$. In this case, the component Pr decreases with increasing $q$ (with decreasing $\lambda_{3}$ ), and the component $P_{3}$ increases. The increase in the component $P_{3}$ is faster than the decrease in the component Pr, Fig. 4.

Option. 2. The repair system provides replacement of the deceased objects from the ready state with an intensity $\lambda_{3}=\omega_{1}$, with simultaneous restoration of objects by repair. Repaired objects are sent to replenish the stock $(\rho=0)$.

To estimate the effectiveness of the use of reserves for this variant, we calculate the numerical values of $R_{\mathrm{g} 2}, \mathrm{R}_{32}, \mathrm{R}_{\mathrm{g} 32}$, and $\eta_{2}$ as a function of the parameter

$\mathrm{K}_{1}=\frac{\omega_{1}}{\mu_{1}}$ The results of calculations are presented in Table. 4 .

From Table 4 it follows that under this variant of resource consumption, the recovery efficiency determined by the gain in the availability indicator $\eta_{2}$ increases with the 
parameter $\mathrm{K}_{1}=\frac{\omega_{1}}{\mu_{1}}$. This is explained by the fact that the indicators $\mathrm{R}_{\mathrm{g} 2}$ and $\mathrm{R}_{\mathrm{G} 3}$ in this case decrease with the decrease of the productivity $\mu_{1}$ much slower than the index $\mathrm{Rg}$, when there is no stock (see Table 1).

Comparing the results of the calculations in Table 3 and Table 4, we can conclude that the parameters of $\mathrm{Pr}$ in both variants vary identically. The indicator $\mathrm{P}_{32}$ decreases and coincides with the exponents. $\operatorname{Pr}_{2}$ and $\mathrm{P}_{32}$. The exponent $\mathrm{R}_{\mathrm{G} 32}$ for the variables $\mathrm{K}_{1}<1$ has a larger value than the exponent $\mathrm{P}_{\mathrm{G} 3}$ for the variables $\mathrm{q}<1$.

Table 4. The results of calculating the indices $\dot{\mathrm{R}}_{\mathrm{g}_{2}}, \mathrm{R}_{\mathrm{z} 2}, \mathrm{R}_{\mathrm{gz}_{2}}$ and the gain $\eta 2$ (variant 2)

\begin{tabular}{|c|c|c|c|c|c|c|c|c|c|}
\hline $\mathrm{K}_{1}$ & 0,00 & 0,10 & 0,20 & 0,30 & 0,50 & 1,00 & 2,00 & 3,00 & 5,00 \\
\hline$\dot{\mathrm{R}}_{\mathrm{g} 2}$ & 0,50 & 0,48 & 045 & 0,43 & 0,40 & 0,33 & 0,25 & 0,20 & 0,14 \\
\hline $\mathrm{R}_{32}$ & 0,50 & 0,48 & 0,45 & 0,43 & 0,40 & 0,33 & 0,25 & 0,2 & 0,14 \\
\hline $\mathrm{R}_{\mathrm{g} 32}$ & 1,00 & 0,95 & 0,91 & 0,87 & 0,80 & 0,67 & 0,50 & 0,40 & 0,27 \\
\hline$\eta_{2}$ & 1,00 & 1,05 & 1,09 & 1,13 & 1,20 & 1,30 & 1,50 & 1,60 & 1,70 \\
\hline
\end{tabular}

A graphic representation of the gains in the productivity of repair organs is given in Fig. 3.

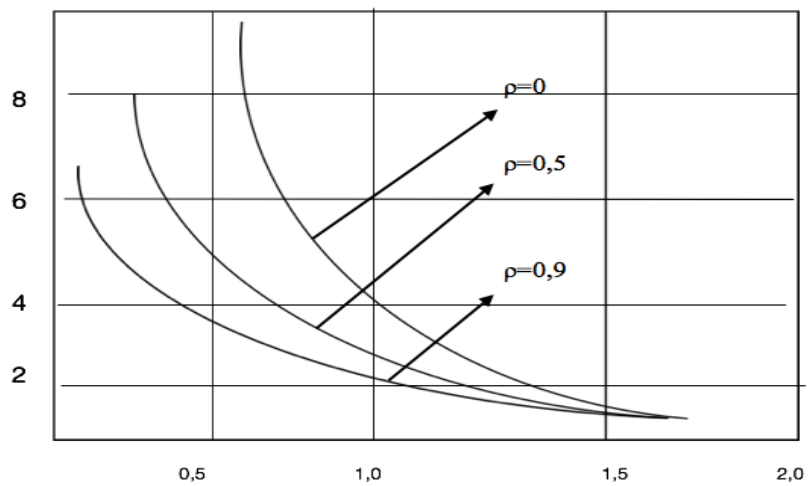

Fig. 3. Dependence of winnings in productivity repair bodies from the ratio $\lambda_{3}$ and $\omega$

Dependence of the gain in the performance of repair organs on the ratio of the change in the readiness indicators $\mathrm{P}_{\mathrm{g} 1}, \mathrm{P}_{\mathrm{g} 2}, \mathrm{P}_{31}, \mathrm{P}_{32}, \mathrm{Pg}_{31}, \mathrm{Rg}_{32}$, depending on the parameters $q=\frac{\omega_{-1}}{\lambda_{3}}$ and $\mathrm{K}_{1}=\frac{\omega_{1}}{\mu_{1}}$ is shown in Fig.4.

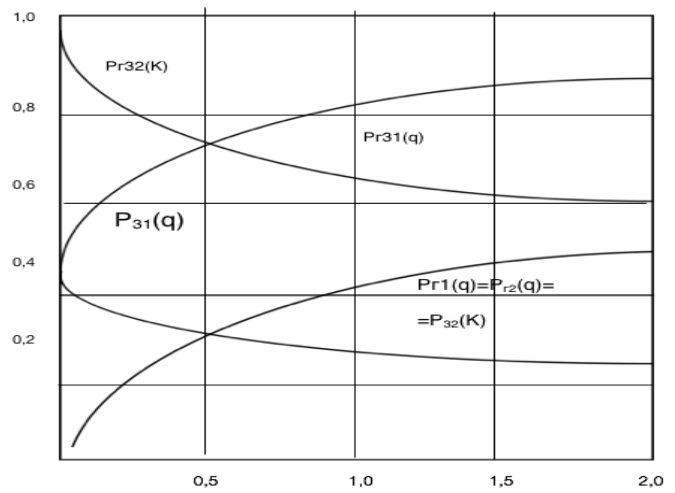

Fig. 4. Dependence of readiness indicators from the parameters K and q for $\rho=0$ 


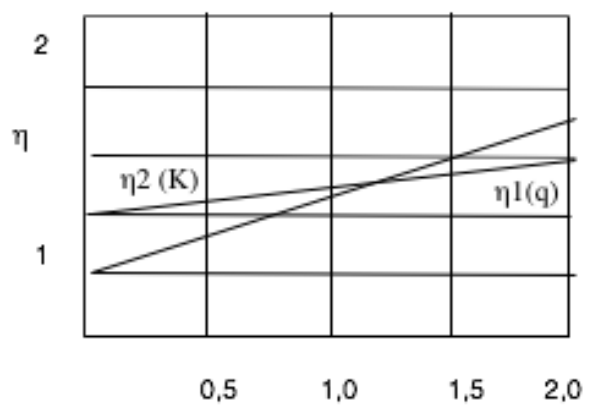

Fig.5. Dependence of winnings in the readiness of objects from strategies for the use of reserves in the repair organs

From Fig. 5, in particular, it follows that $\eta_{1}$ has values greater than one, with the values $q=\frac{\omega 1}{\lambda_{3}}>2 / 3$. Therefore, a repair option with a reserve of objects in which the capacity $\mu$ is equal to the arrival rate of the reworking fund $\omega$ is preferable when $\lambda z<1.5 \omega$, i.e. when the stock is limited.

The gain of $\eta_{2}(\mathrm{~K})$ is always greater than 1. It means that the repair option with a reserve of objects, in which the arrival rate of the reworking fund is equal to the intensity of the stock consumption $\lambda_{3}=\omega$, is always effective, regardless of the value $K_{1}=\frac{\omega_{1}}{\mu_{1}}$ However, $\eta_{2}(K)<$ $\eta_{1}(\mathrm{q})$ for the values of the variables $\mathrm{K}>2 / 3$ which the arrival rate of the reworking fund is equal to the intensity of the stock consumption $\lambda_{3}=\omega$, is always effective, regardless of the value $K_{1}=\frac{\omega_{1}}{\mu_{1}}$ However, $\eta_{2}(K)<\eta_{1}(q)$ for the values of the variables $K>2 / 3$.

\section{Conclusion}

The creation of a reserve of repair facilities can significantly reduce the performance requirements for remorgants. So, if the condition is fulfilled, in which the number of objects that have left for repair is replaced by an equal number of spare objects, the productivity of the repair bodies doubles.

The effectiveness of stock creation increases as the capacity of repair facilities decreases and the share of refurbished facilities returned to operation decreases. Thus, with a ratio in the intensity of expenditure of reserves and the release of objects in repair equal to 0.5 , with a return of repaired specimens at $\rho=0$, the gain in the productivity of repair organs can reach threefold (size) and with an increase of this fraction to 50\% - only twofold

Of the two compared repair strategies using the stock of objects, the readiness of objects in strategy 2 , when $\lambda_{3}=\omega_{1}$ strategy 2 is large when unlimited possibilities of repair organs, when $\mathrm{K}=\frac{\omega_{1}}{\mu_{1}}<1$. Consequently, when there is a small percentage of the failure of the objects or when there are powerful repair organs $\left(\mu_{1}>\omega_{1}\right)$, it is more expedient to use the reserves according to the law $\lambda_{3}=\omega_{1}$.

With limited reserves or with intensive output of objects for repair, strategy I is preferred, in which the performance of the repair bodies should be equal to the rate at which objects return to repair $\left(\mu_{1}=\omega_{1}\right)$.

In order to determine the optimal balance between the stock size and the performance of the repair facilities, it is necessary to take into account economic constraints associated with the relative costs of increasing the productivity of repair bodies or the costs of creating inventories. 


\section{References}

1. A. Barucha-Rid, Elements of the theory of Markov processes (Nauka, M, 1969)

2. G. Fomin. Systems and queuing models in commercial activity: (Finansi and statistika, M. 2000)

3. E. Ventcel, A. Ovcharov, Theory of random processes and its applications. Tutorial (Akademij, M, 2003)

4. A. Mostovskoy. Numerical methods and mathematics. Tutorial. (MGPU, Murmansk, 2009)

5. B. Gnedenko, I. Kovalenko. Introduction to the theory of queuing.(Nauka, 1966)

6. V.Varnikov. Questions of Applied Mathematics and Computational Mechanics, Coll. of Works No 18. The model of the functioning of recovery channels with limited resources. 2015

7. A. Borboni, R. Faglia, Characterization of a new SMA actuator. Procedia Engineering, 87, pp. 1378-1381. (2014) DOI: 10.1016/j.proeng.2014.11.699

8. A. Borboni, R. Faglia, Journal of Applied Mechanics, 80 (2), art. no. 021003, (2013), DOI: $10.1115 / 1.4007721$ 